\title{
EL ÁMBITO DEL DERECHO EN LA DESCENTRALIZACIÓN TERRITORIAL COLOMBIANA Y LA COMUNIDAD ANDINA [CAN] *
}

\section{The field of Law in Colombian territorial decentralization and the Andean Community (CAN)}

Carolina Blanco Alvarado**

Recepción: 5/05/18. Aceptación: 22/11/18

DOI: http://dx.doi.org/10.21017/Rev.Repub.2019.v26.a61

\section{RESUMEN}

La Comunidad Andina (CAN) ha sido considerada como un Organismo Internacional con características diferentes a otros organismos internacionales, motivo por el cual resulta de interés analizar la relación entre los sistemas de administración denominados descentralización territorial colombiano y CAN desde el escenario jurídico, esto es, descartando la perspectiva política, económica y social de la referenciada relación. Para el efecto, es pertinente no olvidar que a la CAN le fueron otorgadas competencias comunitarias supranacionales sustentadas en directrices comunes, provenientes de las voluntades de los gobiernos andinos, con un marco normativo propio.

Es en este sentido que el problema de investigación que se pretende resolver en el presente estudio se circunscribe a analizar el ámbito jurídico de dos sistemas de organización de la administratción como lo son la CAN y la descentralización territorial colombiana. Como respuesta al referenciado problema de investigación se considera que en la actualidad, a pesar de los fenómenos de

* El presente manuscrito es producto del proyecto de investigación Derecho, Estado y Sociedad gestionado en la Universidad Católica de Colombia en el marco del Grupo de Investigación: Derecho Público.

* Doctora en Derecho de la Universidad Santo Tomás (Sede Bogotá). Magíster en Derechos Fundamentales de la Universidad Carlos III de Madrid, España. Especialista en Derecho Constitucional del Centro de Estudios Políticos y Constitucionales de Madrid, España. Especialista en Derecho Administrativo de la Universidad del Rosario, Bogotá. Abogada de la Universidad Externado de Colombia. Docente e investigadora de la Universidad Católica de Colombia. Correo electrónico: Carolinablancoalvarado@ hotmail.com 
globalización, mundialización e internacionalización, se puede evidenciar que no existen las condiciones necesarias para que ambos sistemas de organización funcionen armónicamente desde el escenario jurídico; a pesar de corroborarse competencias concurrentes en el escenario propio de la CAN y de la descentralización territorial colombiana.

Palabras clave: Descentralización territorial, CAN, proceso andino de integración, competencias.

\begin{abstract}
The Andean Community (CAN) has been considered as an International Organization with different characteristics from other international organizations, which is why it is of interest to analyze the relationship between the administration systems called Colombian territorial decentralization and CAN from the legal scenario, that is, discarding the political, economic and social perspective of the referenced relationship. For this purpose, it is important not to forget that the CAN was granted supranational community competencies supported by common guidelines, coming from the will of the Andean Governments, with its own regulatory framework.
\end{abstract}

It is in this sense, that the research problem that is intended to be solved in the present manuscript is limited to analyzing the legal scope of two systems of organization of the administration such as the CAN and the Colombian territorial decentralization. In response to the referenced research problem it is considered that at present, despite the phenomena of globalization, globalization and internationalization, it can be seen that the necessary conditions do not exist so that both systems of organization function harmoniously from the legal scenario; In spite of corroborating concurrent competences in the own scenario of the CAN and the Colombian territorial decentralization.

Key words: Territorial Decentralization, CAN, Andean Integration Process, Competencies.

\title{
INTRODUCCIÓN
}

La vinculación de Colombia al proceso andino de integración plantea reflexiones desde los escenarios económico, político, jurídico y social. Es por ello que a través de las presentes líneas se pretende analizar la repercusión jurídica de la vinculación de Colombia a la CAN desde las competencias descentralizadas territorialmente. 
Para el efecto, es importante resaltar que en el proceso de integración andino opera la transferencia del ejercicio de competencias del Estado colombiano al órgano supranacional denominado CAN y que en el Estado colombiano opera un sistema de organización territorial descentralizado, en el que también opera la trasferencia de competencias desde el poder central a las entidades territoriales colombianas.

El Estado-Nación ha sido objeto de varias trasformaciones, las cuales se materializan de distintas formas; una de ellas es a través del proceso de globalización. A nadie pueden sorprender los cambios coyunturales que ha sufrido el globo terráqueo, los cuales han puesto de manifiesto la crisis del Estado y en modo alguno su incapacidad para adaptarse y asumirlos. En este orden de ideas, reiteradamente la Doctrina colombiana ha manifestado la crisis de la descentralización territorial, entre otros aspectos por factores de orden económico, dejando de lado presupuestos de orden jurídico que no permiten el avance de la descentralización territorial colombiana ${ }^{1}$; es por lo anterior que se considera procedente analizar acuciosamente los elementos de orden jurídico para que la descentralización territorial produzca los efectos deseados antes de contextualizar el escenario económico de dicho sistema de organización administrativo.

En la actualidad, la descentralización territorial ocupa uno de los principales lugares dentro de la agenda de discusión de Colombia; lo anterior en razón a las vicisitudes que dicho sistema de organización administrativo ha evidenciado, y a la necesidad de contextualizar dicho sistema con otros sistemas de organización nacional e internacional. Para el efecto, es importante anotar que analizar la descentralización sin tomar en cuenta las tendencias provenientes de la globalización conduciría a interpretaciones erradas y limitadas; es por ello que resulta de interés analizar a la descentralización territorial colombiana desde la perspectiva del proceso de integración andina, el cual pretende matizar los efectos del fenómeno de la globalización en los Estados andinos.

La temática es pertinente si se toma en cuenta como ejemplo a la Unión Europea, la cual consiguió su constitución de manera simultánea con los procesos de descentralización que vive cada uno de los países que la componen; esto en aras de

1 Al respecto, es importante anotar que «el mundo actual obedece a nuevas circunstancias que exigen una perspectiva igualmente nueva para el Estado y para el derecho administrativo; ello en razón a que se están generando cambios que rompen paradigmas tradicionales. Dentro de los citados paradigmas tradicionales se encuentra el funcionamiento político y jurídico de la descentralización territorial y la integración andina, fenómenos que, más allá del anhelo y la retórica, requieren de caminos que conduzcan a su concreción» (Blanco, 2015, p. 163). 
crear propuestas supranacionales como consecuencia de la globalización ${ }^{2}$ (Quiroz, 2014). Para el efecto, es importante resaltar que el fenómeno de la globalización ${ }^{3}$ exige de manera simultánea internacionalización y localización, lo que ha dado lugar a la glocalización. En otras palabras, lo local, para gestionarse en la actualidad, tiene que internacionalizarse, y lo global, para existir, requiere de espacios estratégicos desde donde proyectarse (Sassen, 1999).

\section{PROBLEMA DE INVESTIGACIÓN}

¿En qué medida y desde qué escenario y perspectiva jurídica, la CAN incide en la descentralización territorial colombiana?

\section{ESTRATEGIA METODOLÓGICA UTILIZADA EN LA INVESTIGACIÓN}

El presente artículo supuso una metodología de investigación cualitativa, ya que pretendió explicar los sistemas de organización administrativa denominados descentralización territorial y CAN, desde el escenario jurídico. El tipo de investigación es de carácter exploratorio en razón a que su objetivo fue examinar un problema de investigación poco estudiado. A la vez es una investigación descriptiva en razón a que pretendió especificar las principales propiedades de la relación entre la CAN y la descentralización territorial colombiana desde el ámbito jurídico.

\section{RESULTADOS}

\section{Las coincidencias de la descentralización territorial colombiana y la CAN}

La CAN ${ }^{4}$ fue creada en 1996, a través del Acuerdo de Cartagena, con personería jurídica internacional, cuya finalidad es promover el desarrollo de los Esta-

2 Se coincide con el criterio de la profesora Insignares, para quien la integración aparece como parte inherente a la globalización (Insignares, 2007).

3 De conformidad con Villamil «la globalización puede comprenderse mejor como un proceso o una serie de procesos, más que como una condición singular. No refleja una simple lógica de desarrollo lineal, ni prefigura una sociedad mundial o una comunidad mundial. Más bien, refleja la aparición de redes y sistemas interregionales de interacción e intercambio» (Villamil, 2016, p. 80).

4 Es importante resaltar que de conformidad con Fariñas Dulce, «la única posibilidad de hacerle frente a la globalización económica es a través de un Estado que encuadre sus políticas dentro de un campo jurídico transnacional o global, ya que hasta el momento 
dos andinos en condiciones de equidad, mediante la integración y la cooperación económica y social. Dicha Organización Internacional está conformada por Bolivia, Colombia, Ecuador y Perú, y tiene tres características fundamentales: la existencia de un orden jurídico andino, la supranacionalidad, y la creación de órganos comunitarios independientes y autónomos $^{5}$ (Rosell, 1999).

La descentralización territorial ha sido entendida como el sistema de organización administrativo a través del cual el poder central transfiere competencias a órganos subnacionales o entidades territoriales para que estos las gestionen con autonomía e independencia.

Los elementos de configuración propios de la CAN y de la descentralización territorial son similares. Para el efecto, las competencias asignadas a la CAN ${ }^{6}$ y a las entidades descentralizadas territorialmente han sido asignadas mediante ley. De otro lado, los referidos sistemas de organización administrativos deben funcionar en Estados democráticos. Adicionalmente, al estudiar y reflexionar sobre los citados sistemas se observa la preponderancia del ejecutivo, a través del Gobierno central como protagonista tanto de la descentralización territorial como del proceso andino de integración. La similitud también se corrobora por la transferencia del ejercicio de competencias que se da tanto en la descentralización territorial como en el proceso andino de integración. En todo caso, la similitud más relevante entre estos fenómenos está en que a ambos sistemas de organización se les ha ordenado la gestión de competencias concurrentes. Para el efecto, es importante resaltar que competencias relacionadas con el transporte, la educación, la salud, el medio ambiente y el turismo fueron asig-

el derecho internacional ha sido insuficiente para lograr compaginar las exigencias de la actual sociedad internacional» (Fariñas, 2004). Lo anterior permite resaltar la importancia del proceso andino de integración como instrumento capaz de hacer jaque al fenómeno de la globalización frente a los Estados andinos.

5 De conformidad con Blanco (2014), a pesar de que la Comunidad Andina se ha constituido en el proceso de integración más antiguo de Suramérica, reiteradamente se ha manifestado la crisis del mismo. Para efectos de lo anterior se considera que a pesar de que el núcleo esencial de cualquier proceso de integración es económico, las causales que obstaculizan a la CAN son de carácter jurídico y político. Dicha afirmación supone que la crisis de la citada organización internacional obedece a la falta de capacidad jurídica y política de los países miembros de comportarse en el ambiente propio de un proceso de integración (Blanco, 2014).

6 Es de importancia resaltar que el Acuerdo de Cartagena por el cual se crea a la CAN ha sido considerado como el cimiento de la "construcción integracionista» de los países andinos, el cual puede seguir adelante si se adopta una visión finalmente integradora (Blanco, 2013 a). 
nadas tanto a la CAN7 como por la descentralización territorial (Patarroyo \& Benavides, 2014).

Es por lo anterior que resulta procedente afirmar la concurrencia entre las nociones de integración andina y descentralización territorial. La citada afirmación presupone la modificación del papel en el mundo contemporáneo de las entidades territoriales.

\section{El ámbito jurídico de la descentralización territorial colombiana y la CAN: Propuesta de Avance}

La integración andina es un proceso en virtud del cual los gobiernos andinos han adoptado con el apoyo de instituciones comunes, decisiones conjuntas en aras de intensificar su interdependencia y generar beneficios económicos, políticos y jurídicos comunes. El concepto de integración andina no se circunscribe a la cooperación internacional; para el efecto, la profesora Insignares ha considerado que «su significado pleno es el de la concertación deliberada, entre varias unidades autónomas, de un programa de acción conjunta hasta su culminación, es decir, hasta conseguir la integración perseguida, para lo cual crean una organización independiente, con esa finalidad específica, dotándola de poderes y recursos suficientes para lograrla. Implica, pues, interdependencia entre iguales, un obrar en conjunto, una reciprocidad de intereses, una comunidad que no sacrifica la pluralidad »(Insignares, 2007, p. 45).

Dialogar sobre la integración andina supone no olvidar que frente a dicho fenómeno el Estado colombiano mantiene la titularidad de la soberanía, sin perjuicio de lo cual puede, si sus intereses y conveniencia lo aconsejan, transferir a la CAN la administración práctica de ciertos campos de su actividad (Perotti, 2007). En otras palabras, la CAN no es un órgano internacional que desplace a los Estados miembros porque los mismos, al suscribir el Tratado Constitutivo, han acordado limitar el ejercicio de sus competencias, y transferir el ejercicio de algunas competencias a dicha Organización Internacional, lo cual permite la existencia de la supranacionalidad, la cual debe ser comprendida como el ejercicio en común de competencias soberanas, y no como el abandono de parte de la soberanía estatal ${ }^{8}$ (Palomares \& Calonje, 2015).

7 Blanco ha considerado que en la actualidad emitir una definición exacta del término integración, en razón a que el manuscrito desarrolla temáticas propias del proceso de integración andino, «es un aspecto necesario, pero difícil; es por ello, que esta ha sido enfocada desde diferentes puntos de vista. En este sentido, existe un alto grado de confusión en el mundo acerca de lo que debe entenderse por integración, entre otras causas porque el fenómeno es realmente reciente y por tanto la teoría acerca de él no está agotada» (Blanco, 2013 b, p. 95).

8 En el mismo sentido ha señalado la Corte Constitucional que el concepto de supranacionalidad genera que los países miembros transfieran, a un organismo internacional, 
Es por lo anterior que resulta pertinente afirmar que el Derecho administrativo en la actualidad no solamente está sometido por las normas de orden legal, sino también por normas de orden comunitario (Dromi, 2003); y en este orden de ideas el Derecho Comunitario ha procedido en modo alguno con la transformación del Derecho Administrativo colombiano. Lo anterior obedece fundamentalmente a que en la actualidad se evidencian Decisiones y Resoluciones emitidas por la CAN, que tenían origen en autoridades estatales colombianas, y que ahora se originan en instancias propias de la CAN. Adicionalmente, algunas decisiones emitidas por autoridades estatales colombianas son controladas jurisdiccionalmente por la CAN, a través del Tribunal Andino de Justicia.

Lo dispuesto en el párrafo anterior permite afirmar que el bloque de legalidad para resolver problemas jurídicos en el Estado colombiano se ha ampliado como consecuencia de las reglas y principios provenientes de la normatividad comunitaria, lo cual genera para las autoridades internas colombianas, en el marco de sus competencias, no solo dar cumplimiento a las normas nacionales, sino también a las normas comunitarias (Rodríguez, 2010).

En el mundo contemporáneo se ha corroborado la incapacidad de la norma emitida por los legislativos de los Estados para regular la globalidad de los hechos que plantean contextos cada vez más cambiantes e inciertos (Núñez, 2009). Es por esto que el Estado era el principal protagonista de la producción de normas. El escenario en virtud del cual los legislativos eran los únicos órganos competentes para legislar, en la actualidad se está matizando por el impacto de la globalización en la estructura estatal. Lo dispuesto anteriormente se hace evidente cuando el profesor Vélez conceptúa sobre el fenómeno de la descentralización en la actualidad así: «con la descentralización como estrategia, surge una nueva forma de gobernar, que expresa una clara tendencia a transferir el poder hacia dos direcciones opuestas: hacia arriba, a los organismos supranacionales ${ }^{9}$, y hacia abajo, a núcleos más pequeños, para descentra-

determinadas atribuciones nacionales que permiten que dicho órgano expida normas con vocación de integrar el derecho interno de cada uno de los Países Miembros (Sentencia C-331/96). En este sentido, la Corte Constitucional prevé que la adquisición de poderes reguladores por los organismos comunitarios proviene de una transferencia de competencias que se gestiona de manera voluntaria e inicialmente, en el tratado constitutivo, lo que permite una tranferencia de lo nacional a lo supranacional.

9 Al respecto, la Corte Constitucional, en sentencia C-137 de 1996, señaló:

"Como es sabido, el concepto de supranacionalidad -dentro del que se inscribe el Acuerdo de Cartagena-implica que los Países Miembros de una organización de esta indole se desprendan de determinadas atribuciones que, a través de un tratado internacional, son asumidas por el organismo supranacional que adquiere la competencia de regular de manera uniforme para todos los Países Miembros sobre las precisas materias que le han sido transferidas, con miras a lograr 
lizar la toma de decisiones. El objetivo, en ambos casos, es acercar el gobierno a los gobernados, dar mayor legitimidad al sistema y responsabilizar a los ciudadanos en la toma de decisiones que afectan su propio destino» (Vélez, 2003, p. 49).

Atendiendo lo dispuesto por el profesor Vélez, se puede concluir que el fenómeno de la descentralización territorial ya no es un aspecto de importancia única y exclusiva por el Estado colombiano, sino que dicho fenómeno empieza a cobrar importancia en el escenario exterior, y para efectos de estas líneas, en el escenario supranacional, esto es, en el marco del proceso de integración andino.

La CAN tiene una extensión territorial de 4־718.000 kilómetros cuadrados y una población que supera los 110 millones de habitantes (Blanco, 2014). Esto supone una significativa importancia en América Latina. Sin embargo, los aportes e impactos de la CAN en los Estados miembros -que podrían ser mayoresse han visto disminuidos por factores de orden político, jurídico y económico (Rodríguez, 2016).

Es en este sentido que a través de las presentes líneas se pretende proponer algunos presupuestos de orden jurídico necesarios para que la CAN y la descentralización territorial colombiana puedan funcionar coordinadamente, y de esta manera lograr el avance del proceso andino de integración y de la descentralización territorial colombiana.

1. La configuración de necesidades locales compatibles con las competencias que a la fecha gestiona el proceso andino de integración (Vidal, 1975). En otras palabras, para que la descentralización territorial colombiana pueda funcionar en el marco de la CAN, es necesario que existan necesidades locales que sean compatibles con las competencias propias de la CAN.

2. La garantía de la autonomía administrativa a las entidades territoriales. La descentralización territorial supone un control de tutela por parte del

procesos de integración económica de carácter subregional. Las normas supranacionales despliegan efectos especiales y directos sobre los ordenamientos internos de los Países Miembros del tratado de integración, que no se derivan del común de las normas de derecho internacional. Por una parte, esta legislación tiene un efecto directo sobre los derechos nacionales, lo cual permite a las personas solicitar directamente a sus jueces nacionales la aplicación de la norma supranacional cuando esta regule algún asunto sometido a su conocimiento. En segundo lugar, la legislación expedida por el organismo supranacional goza de un efecto de prevalencia sobre las normas nacionales que regulan la misma materia y, por lo tanto, en caso de conflicto, la norma supranacional desplaza (que no deroga) -dentro del efecto conocido como preemption- a la norma nacional.» (Corte Constitutional, 1996). 
poder central, aspecto que es completamente entendible en un Estado Unitario como el colombiano. Sin embargo, en el marco del proceso andino de integración es de relevancia que el citado control de tutela permita cierto grado de autonomía para que las entidades descentralizadas territorialmente puedan actuar frente a la CAN. Al respecto, coincidimos con el profesor Penagos, para quien «La verdadera descentralización exige eliminar las políticas paternalistas, las limitaciones impuestas por el poder central, la coordinación y las políticas centralistas que desconocen la autonomía» (Penagos, 2004, p. 41). A pesar de lo dispuesto por el profesor Penagos, se observa en la actualidad la existencia de un centralismo en el sistema de organización administrativo del Estado colombiano, el cual ha estado presente desde el momento de la expedición de la carta política de 1991 (Betancur, 2013, p. 194).

El artículo primero de la Carta Política reconoce la autonomía ${ }^{10}$ de las entidades territoriales como elemento integrante de una República unitaria y descentralizada. En este sentido, se entiende por autonomía administrativa de las entidades territoriales, el ámbito de intereses propios y el reconocimiento de que esos intereses propios han de ser normados y regidos por entes específicos bajo la dirección y supervisión del Estado (Suelt, 2008). Es en este sentido que resulta procedente lo afirmado por el profesor Penagos, quien considera que «la eficacia de la descentralización depende de la conciliación de la autonomía propia del Estado unitario con la autonomía que proclama la descentralización» (Penagos, 1996, p. 5). Lo dispuesto en líneas anteriores supone, sin lugar equívocos, la armonización de la autonomía propia de las entidades descentralizadas y del Estado unitario.

3. Claridad normativa en las competencias asignadas a las entidades territoriales descentralizadas ${ }^{11}$. Es importante que la normatividad que prevé las competencias de las entidades territoriales evidencie límites competenciales $^{12}$, evite la duplicación en la asignación de competencias y

$10 \mathrm{Al}$ respecto coincidimos con el profesor Suelt, para quien dicha autonomía «no se agota en la dirección política de las entidades territoriales, sino que estas deben además gestionar sus propios intereses, lo que permite concretar su poder de dirección administrativa» (Suelt, 2008).

11 En la actualidad existe un proceso gradual de desregulación en la temática propia de la garantización en la prestación de servicios públicos, en especial de los servicios públicos asignados a las entidades territoriales (Servicio, 2005).

$12 \mathrm{Al}$ respecto es importante anotar que las competencias en el plano local aumentan, sea por el incremento de las demandas en ese ámbito, por la solicitud de los gobiernos locales o por los traspasos de los órganos centrales. Sin embargo, este crecimiento no tiene sentido si el órgano correspondiente no tiene capacidad de asumirlas (Carrion, 2003, p. 31). 
competencias tan generales que en la práctica resultan impracticables o no concretan ninguna competencia en particular. Lo dispuesto anteriormente es de suprema relevancia para efectos de asignar adecuadamente responsabilidades a las correspondientes entidades territoriales. Ejemplo de lo establecido en líneas anteriores se encuentra cuando el ordenamiento jurídico colombiano prevé que una de las atribuciones de las entidades territoriales es la de ejercer las competencias que le correspondan; dicha competencia es absolutamente ambigua y etérea, ya que las competencias de las entidades territoriales no han sido claramente definidas en el marco legal y constitucional. Las pocas competencias referenciadas en la Constitución colombiana están supeditadas a lo que el legislador reglamente sobre el particular (Eraso, 2004).

4. Eficiencia en los principios rectores de la descentralización territorial; esto es de principios como la subsidiariedad, la coordinación y la concurrencia. De conformidad con el principio de subsidiariedad, el gobierno central no debe intervenir en las competencias que a las entidades territoriales por vía legal o constitucional les hayan sido atribuidas; y en especial aquellas que resulten concomitantes con las competencias de la CAN. A diferencia del principio de subsidiariedad, el principio de coordinación supone la toma de decisiones conjuntas por parte de del jefe de Estado y de Gobierno, las entidades territoriales y los ministros. El principio de concurrencia pretende asegurar participación activa de las entidades territoriales en las decisiones que considere procedentes el Gobierno central. Lo anterior en razón a que la concurrencia no puede significar imposición de hecho ni de derecho, en la gestión de las competencias para la concreción de los intereses de la descentralización territorial y del proceso andino de integración (Barragán, 2016).

5. La identificación en el Derecho Interno colombiano del grado de descentralización y de autonomía de las entidades territoriales. Lo anterior en razón a que tanto la descentralización territorial colombiana, como la autonomía de las entidades territoriales son cuestión de grado (Maldonado, 2008). Al respecto es importante resaltar que la denominación que efectúa la Carta Política, identificando al Estado colombiano como república unitaria, descentralizada y con autonomía de sus entidades territoriales, no significa nada respecto del grado de descentralización y de autonomía. La no identificación en el Derecho Interno colombiano del grado de descentralización y de autonomía de las entidades territoriales genera como consecuencia lagunas legislativas cuando se quiere identificar clara y expresamente el nivel de autonomía y de descentralización en las competencias de las entidades territoriales. 
6. Existencia de recursos económicos. En aras de que la descentralización territorial colombiana funcione en el proceso de integración andino, es necesaria la existencia de recursos económicos ${ }^{13}$ que permitan gestionar de manera concurrente las competencias de las correspondientes entidades territoriales y de la CAN. Lo anterior supone por parte del Gobierno Nacional la reserva de los respectivos recursos en el plan nacional de desarrollo y en el presupuesto anual general del Estado colombiano.

Es importante por parte del Gobierno Central, garantizar, promover y fortalecer las transferencias municipales y aumentar la capacidad de generación de recursos propios por parte de las entidades territoriales; lo cual genera como obligación a las mismas que las autoridades territoriales demuestren racionalidad en la gestión del gasto público y eviten el sobreendeudamiento y el despilfarro.

La CAN permite la posibilidad de obtener ingresos por parte de las entidades territoriales descentralizadas, lo cual justifica los gastos de las mismas. Al respecto, es importante no olvidar lo dispuesto por el profesor Perry, quien considera que «las entidades territoriales que logran financiar gran parte de su gasto con contribuciones propias, evidenciaran mayor estabilidad económica» (Perry, 1999). En concordancia con lo dispuesto por el profesor Perry, el profesor Nickson considera que «si las entidades subnacionales ejercen importantes funciones de gasto, también deberían tener mayores responsabilidades en la obtención de ingreso»(Nickson, 1998, p. 32). Esto permite afirmar que los gobiernos subnacionales gestionan mejor sus competencias cuando la obtención de sus ingresos y la de sus gastos se relacionan directamente (Bird, 1999).

7. Normas constitucionales que permitan dinamizar el Derecho Derivado Comunitario Andino. Esto obedece a que no es asimilable el tratado constitutivo de la CAN a la Carta Política colombiana, ya que quienes elaboran el citado tratado constitutivo son los Estados miembros (Fondevilla, 2009). La existencia de normas constitucionales que permitan dinamizar el Derecho Derivado Comunitario Andino permite justificar desde el marco constitucional la transferencia del ejercicio de competencias soberanas del Estado colombiano a la CAN y así evitar en modo alguno, problemas jurídicos de contradicción entre el Derecho Comunitario Andino y Ordenamiento Jurídico colombiano.

13 Al respecto, el profesor Carrión considera que «Es necesario determinar los mecanismos adecuados para la transferencia y la oportunidad de los recursos, eliminar la discrecionalidad (que fortalece el clientelismo y la corrupción) y no estimular la dañina pereza fiscal» (Carrión, 2003, p. 32). 
8. Armonización y articulación del Derecho Comunitario Andino con el Derecho Interno. En aras de que la descentralización territorial colombiana funcione en el marco del proceso andino de integración, es indispensable que desde el Gobierno Central se cumpla de manera eficiente y eficaz los postulados propios del Derecho Comunitario Andino o Derecho de la Integración ${ }^{14}$; esto es, la supranacionalidad que supone el citado sistema jurídico, la primacía o prevalencia ${ }^{15}$ del ordenamiento jurídico andino, el reconocimiento de la existencia del Derecho Comunitario Andino en el Derecho Interno y la aplicabilidad directa ${ }^{16}$ de las normas comunitarias.

\section{CONCLUSIONES}

En el marco de los Estados andinos, es evidente que como consecuencia de la globalización, la noción de soberanía estatal se cuestiona, circunstancia que genera incertidumbre respecto del papel de los órganos internos estatales y su eficiencia para resolver problemas globales. Por lo anterior, se hace pertinente un replanteamiento de la noción de soberanía, y como consecuencia de ello, el análisis dentro de la noción de soberanía, de la injerencia de organismos internacionales supranacionales y comunitarios como la CAN en algunas de las competencias de los Estados andinos.

Es evidente la relación jurídica que existe entre los sistemas de organización administrativos llamados descentralización territorial y CAN. Esto principalmente porque, de conformidad con el marco normativo, se puede corroborar competencias concurrentes en ambos sistemas, lo que supone coordinación entre dichas formas de organización. Lamentablemente, a la fecha, se puede evidenciar que la citada coordinación no existe, entre otros

14 De conformidad con Insignares, «El derecho comunitario andino se convierte en un obstáculo para la integración en la medida en que no se aplique adecuadamente el concepto de supranacionalidad y preeminencia frente a las normas internas del respectivo país miembro.» (Insignares, 2007, p. 34).

15 Al respecto, Núñez considera que «La prevalencia muestra el procedimiento a seguir cuando se genera contradicción entre una norma comunitaria andina y una interna. En esta situación, no necesariamente debe derogarse la interna, pero por su carácter prevalente se aplica la norma jurídica comunitaria» (Núñez, 2006, p. 28).

16 Al respecto, Núñez considera que la aplicación directa de las normas comunitarias supone que «las decisiones del Consejo Andino de Ministros de Relaciones Exteriores, de la Comisión de la Comunidad Andina y las resoluciones de la Secretaría General serán implementadas y producirán efectos en los países miembros a partir de la fecha de publicación en la Gaceta Oficial del Acuerdo de Cartagena» (Nuñez, 2006, p. 28). 
factores por la misma crisis del fenómeno de la descentralización territorial colombiana y de la CAN.

La vinculación del Estado colombiano al proceso andino de integración exige la implementación en el Derecho Interno, del sistema jurídico Comunitario o Derecho Andino de la Integración, el cual ostenta características especiales y diferentes frente al Derecho Internacional y al Derecho Interno.

Es importante resaltar que de conformidad con el Tratado Internacional denominado Acuerdo de Cartagena y demás protocolos modificatorios, la normatividad comunitaria es obligatoria y prevalence con relación a las normas nacionales. En consecuencia, los funcionarios, de la rama legislativa, la ejecutiva y la judicial deben fundamentar sus decisiones atendiendo al Derecho Interno pero contextualizándolo en el marco de la naturaleza jurídica del Derecho Comunitario. En concordancia con lo dispuesto anteriormente, es de relevancia no olvidar que el Derecho Comunitario Andino es autónomo, de aplicación inmediata, de efecto directo, y con eficacia prevalente; y como consecuencia de ello, Colombia debe abstenerse de emitir medidas que obstaculicen la eficacia del Derecho Comunitario Andino, ya sea que estas provenga de las ramas legislativa, judicial o ejecutiva.

Atendiendo lo dispuesto en líneas anteriores, resulta procedente afirmar que las competencias de la CAN se verán limitadas en la medida en que el Derecho Interno restrinja su supremacía o aplicación directa.

La descentralización territorial colombiana sigue siendo gestionada en el ámbito local, lo cual le resta a la CAN la posibilidad de enfrentar los efectos de la globalización en los Estados miembros y de proponer beneficios a las entidades descentralizadas territorialmente que quieran gestionar conjuntamente algunas de sus competencias con el proceso andino de integración.

La relación jurídica de la CAN y la descentralización territorial colombiana es de interdependencia en razón a que el sistema de organización administrativo descentralizado en la actualidad, y como consecuencia del Derecho Comunitario Andino, debe generar el cumplimiento del Derecho Interno y del Derecho Comunitario Andino.

Para el logro de los objetivos de la CAN es necesario por parte de los países miembros, armonizar las políticas jurídicas, económicas y sociales, lo cual supone en primera instancia superar las vicisitudes de orden jurídico del Derecho Comunitario Andino. 


\section{REFERENCIAS}

Barragán, D. (enero-junio, 2016) La construcción de la mentalidad democrática como necesidad en el posconflicto. Revista Via Inveniendi et Iudicandi, 11(1), 37-57.

Betancur, L. (2013). Descentralización por asociatividad en el nuevo contex to de la organización territorial colombiana. Bogotá, Colombia: Universidad del Rosario.

Bird, R. (1999). Subnational revenues. Annual World Bank, Conference on Development in Latin America and the Caribean, Valdivia (Chile). Memorias Seminario Globalización Territorial gestionado por el Parlamento Andino en Bogotá, Colombia.

Blanco, C. (2015). La influencia positiva de la CAN en la descentralización territorial colombiana. Revista Opinión Jurídica, 14(27), pp. 161-174 Documento extraído el 26 de junio de 2018 de https:/ / revistas.udem.edu.co/index.php/opinion/article/ view/1204/1159

Blanco, C. (julio-diciembre, 2014). La crisis de la Comunidad Andina. Revista de Derecho, 42, pp. 1-32 Documento extraído el 26 de junio de 2018 de http:// rcientificas.uninorte.edu.co/index.php/derecho/article/view/6788/6604

Blanco, C. (enero-junio, 2013 a). La Comunidad Andina en el marco de lo jurídico y de lo político. Revista Prolegómenos-Derechos y Valores, 16(31):173-178. Documento extraído el 26 de junio de 2018 de https://revistas.unimilitar.edu.co/index.php/ dere/article/view/726/479

Blanco, C. (julio-diciembre, 2013 b). Aproximación a la noción de soberanía estatal en el marco del proceso andino de integración, pp. 91-103. Revista Republicana, 15. Documento extraído el 26 de junio de 2018 de http:/ / ojs.urepublicana.edu.co/ index.php/revistarepublicana/article/view/23/21

Carrión, F. (2003). La descentralización en América Latina: una perspectiva comparada. Procesos de descentralización en la Comunidad Andina, Quito, Ecuador: Flasco.

Dromi, R. (2003). Sistema de Derecho Administrativo. Funciones. Documento extraído de http:/ / vlex.com/vid/37725667 el 21 de septiembre de 2011.

Fariñas, M. (2004). Globalización, ciudadanía y derechos humanos. Cuadernos Bartolomé de las Casas. Madrid, España: Editorial Dykinson.

Eraso, Y. (diciembre, 2004). El fracaso de la autonomía territorial esbozada en la Constitución de 1991. Revista Criterio Jurídico, 4, pp. 34-49 Documento Extraído el 28 de febrero de 2013 de http:/ / vlex.com/vid/ 43931974. 
Fondevilla, M. (julio, 2009). La disolución de la soberanía en el ámbito estatal: los efectos de la integración europea. Revista de Estudios Constitucionales, 1, pp. 5677. Documento extraído el 10 de junio de 2013 de http:/ / vlex.com/vid/ 64854169

Insignares, S. (julio, 2007). El derecho comunitario andino ¿obstáculo en la integración? Revista de Derecho de la División de Ciencias Jurídicas, 27.

Maldonado, C. (2008). Alcance de la autonomía territorial en materia de distribución de competencias entre la nación y las entidades territoriales. Madrid, España: Instituto Ortega y Gasset y la Universidad Complutense.

Núñez, M. (2009). La crisis del Estado en Estado, Derecho y Religión en Oriente y Occidente. Documento extraído el 29 de diciembre de 2012 http:/ / vlex.com/vid/ 65576105

Palomares, J. \& Calonje, N. (2015). Tratados de libre comercio Colombia-Asia: Cuestión preliminar y perfiles de negociación. Revista IUSTA, 43(1), 17-41.

Patarroyo, S. \& Benavides, P. (2014). Rupturas Asignificantes: Revisiones críticas en torno al derecho. Revista Via Inveniendi et Iudicandi, 9(1), 7-31.

Penagos, G. (2004). La Descentralización en el Estado Unitario. Bogotá, Colombia.: Ediciones Doctrina y Ley Ltda.

Penagos, G. (1996). Entidades Descentralizadas. Bogotá, Colombia: Ediciones Librería del Profesional.

Perotti, A. (2007). Habilitación Constitucional para la Integración Comunitaria. Buenos Aires, Argentina: Jurua.

Perry, G. (1999). Macroeconmic management in decentralized democracies. Trabajo presentado a Annual WorldBank Conference on Development in Latin American and the Caribbean, Valdivia. Memorias Seminario Globalizaición Territorial gestionado por el Parlamento Andino en Bogotá, Colombia.

Quiroz, M. (2014). Acercamiento a las «oposiciones paradigmáticas» entre neoconstitucionalismo y positivismo jurídico. Revista IUSTA, 141(2), 77-97.

Rodríguez, J. (2010). El Derecho Administrativo frente a la internacionalización del Derecho y en particular frente al Derecho Comunitario. Documento extraído el 20 de diciembre de 2010 de http:/ / vlex.com/vid/77329889

Rosell, M. (1999). Marco institucional y seguridad jurídica en la Comunidad Andina. Lima, Perú: Secretaría General de la Comunidad Andina. 
Sassen, S. (1999). La ciudad global. Buenos Aires, Argentina: EUDEBA, Buenos Aires.

Servicio. (diciembre, 2005). Servicio público y derecho comunitario europeo. Revista catalana de derecho público, 31, pp. 78-90. Documento extraído el 13 de julio de 2013 de http://vlex.com/vid/61476841

Suelt Cock, V. (junio, 2008). La organización territorial colombiana frente a la tendencia contemporánea de configuración federal. Revista de Estudios Socio-Jurídicos, 10-1, 159-200. Documento extraído el 19 de julio de 2013 de http:/ / vlex.com/vid/ 51340715 .

Vélez, R. (2003). Posibilidades de una ley marco de descentralización. Procesos de descentralización en la Comunidad Andina, Quito, Ecuador: Flasco.

Vélez, R. (2006). La inevitable fractura de la normativa andina tras la implementación del TLC en Tratado de Libre Comercio. La integración comercial y el derecho de los mercados. Quito, Ecuador: Universidad Andina Simón Bolívar.

Vidal, J. (1975). Derecho Administrativo. Bogotá, Colombia: Universidad de los Andes.

Villamil, S. (julio-diciembre 2016). Integración como fuente de conflictividad. Choque entre Hegemonía y Cooperación. Revista NOVUM JUS, 10(2):98-120 